April 27, 2022

\title{
Infimum Microlensing Amplification of the Maximum Number of Images of $n$-point Lens Systems
}

\author{
Sun Hong RhIE \\ Institute of Geophysics and Planetary Physics, Lawrence \\ Livermore National Laboratory, Livermore, CA 94550
}

\begin{abstract}
The total amplification of a source inside a caustic curve of a binary lens is no less than 3 . Here we show that the infimum amplification 3 is satisfied by a family of binary lenses where the source position is at the mid-point between the lens positions independently of the mass ratio which parameterizes the family. We present a new proof of an underlying constraint that the total amplification of the two positive images is bigger than that of the three negative images by one inside a caustic. We show that a similar constraint holds for an arbitrary class of $n$-point lens systems for the sources in the 'maximal domains'. We introduce the notion that a source plane consists of graded caustic domains and the 'maximal domain' is the area of the source plane where a source star results in the maximum $n^{2}+1$ images. We show that the infimum amplification of a three point lens is 7 , and it is bigger than $n^{2}+1-n$ for $n \geq 4$.
\end{abstract}

subject headings: gravitational lensing - planetary systems - stars: binaries: general

Submitted to Astrophysical Journal Letters 


\section{Introduction}

Gravitational lensing has been known for three quarters of a century and has been extensively used in studies of quasars and clusters of galaxies. In these cases, the lenses are compound lenses consisting of extended objects such as galaxies and clusters of galaxies. The recent gravitational microlensing experiments looking for baryonic dark matter (Alcock, et al., 1993, Aubourg, et al., 1993, Udalski, et al., 1993) study nearby stars $(\lesssim 50 \mathrm{kpc})$ as source stars, however, and the lenses are point lenses. They are mostly single lenses but some of them have turned out to be binary lenses (Udalski, et al., 1994, Bennett, et al., 1995, Alcock, et al., 1995, Alard, et al., 1995). The detection rate of binary lenses is expected to be about $10 \%$ according to Mao and Paczyński (1991). One interesting class of binary lenses is that of planetary systems, and there is an effort to look for extra solar earth mass planets using gravitational microlensing (Tytler et al., 1995). Obviously, the observational advantage of having to deal with point lens systems is that the simplicity allows high precision experiments. From a theorist's point of view, the current and proposed microlensing experiments have brought a necessity to study fine details of the cleanest lens systems, which in turn would lead to a more sophisticated understanding of more uncertain lens systems.

It is well known that when two masses are nearby, they produce a gravitational lens with a rich structure that depends on the mass ratio and separation between them and the lens structure is best represented by the caustics. The caustic curves are closed cuspy loops, and neither do they self-intersect nor nest each other. (If $n \geq 3$, the lenses are general enough to feature self-intersections and nesting of the caustic loops. See figure 1 for an example of a ternary lens.) A caustic curve of a binary lens defines an inside and an outside and the number of images of a source is five inside a caustic loop and three outside. Infinity lies in the outside domain and the number of the images of a source at infinity is three, one at the source position and the other two at the lens points. Obviously, the source at infinity is unamplified and the total microlensing amplification $A_{\text {tot }}(\infty)=1$. The amplification of the image at the source position is one and that of the image at each lens position is zero. The amplification of a positive image (三 an image with positive parity) is always no less than 1 , and the total amplification $A_{\text {tot }} \geq 1$ in the outside domain. In an inside domain, a source produces two positive images and hence $A_{\text {tot }}>2$. In fact, $A_{\text {tot }} \geq 3$ inside a caustic as shown recently by Witt and Mao (1995). Their main result was the following relation.

$$
\sum_{\text {images }} \frac{1}{J}=1,
$$


where $J$ is the Jacobian determinant of the lens equation. In order to demonstrate (1.1), the authors converted the binary lens equation inside a caustic into a fifth order polynomial equation of $J^{-1}$ using an algebraic computational package Mathematica. They also made an empirical suggestion that there is only one binary lens configuration that accommodates the infimum amplification 3 inside a caustic.

Here we show that there are actually one-parameter family of binary lenses where $A_{\text {tot }} \geq 3$ is saturated inside a caustic. We also present a simple algebraic proof of (1.1) and show that a similar "sum rule" holds in the "maximal domains" of arbitrary $n$-point lens systems. This leads to a necessity to look at the source planes as consisting of graded caustic domains $\left\{\mathcal{D}^{m}\right\}$. In section 4, we discuss the hierarchical structure of the source plane of an arbitrary $n$-point lens and infimum microlensing amplifications of the sources in the "maximal domains."

\section{Inside the Caustics of Binary Lenses}

A lens equation is a mapping from an image position to a source position on the lens plane transverse to the line of sight. If we set the distance scale of the lens plane by the Einstein ring radius of the total mass, the normalized lens equation is given by (Bourassa, Kantowski and Norton, 1973)

$$
\omega=z-\frac{\epsilon_{1}}{\left(\bar{z}-x_{1}\right)}-\frac{\epsilon_{2}}{\left(\bar{z}-x_{2}\right)},
$$

where $z$ and $\omega$ are the image and source positions in complex coordinates, $x_{1}$ and $x_{2}$ are the lens positions on the real axis, and $\epsilon_{1}$ and $\epsilon_{2} \quad\left(\epsilon_{1}+\epsilon_{2}=1\right)$ are the fractional masses of the lens elements. The Jacobian matrix elements of the lens equation (2.1) are

$$
\partial_{z} \omega=1 \quad, \quad \partial_{\bar{z}} \omega=\frac{\epsilon_{1}}{\left(\bar{z}-x_{1}\right)^{2}}+\frac{\epsilon_{2}}{\left(\bar{z}-x_{2}\right)^{2}} \equiv \bar{\kappa},
$$

and the Jacobian determinant is $J=\left|\partial_{z} \omega\right|^{2}-\left|\partial_{\bar{z}} \omega\right|^{2}=1-\left|\partial_{\bar{z}} \omega\right|^{2} \leq 1$. Lensing is an inverse mapping of the lens equation and the inverse Jacobian matrix elements are given by

$$
\partial_{\omega} z=\frac{1}{J} \quad, \quad \partial_{\bar{\omega}} z=-\frac{\bar{\kappa}}{J} .
$$

For a positive image, $0 \leq J \leq 1$ and the amplification $A=|J|^{-1} \geq 1$. A source inside a caustic has two positive images, and the total amplification of the positive images $A_{+} \geq 2$. Therefore, combined with (1.1), the total amplification $A_{\text {tot }} \geq 3$. 
In order to find all the lens parameters where the inequality $A_{\text {tot }} \geq 3$ is saturated, we note that the minimum $A_{\text {tot }}=3$ can occur only if both of the positive images have $J=1$ ( or $\partial_{\bar{z}} \omega=0$ ). They are the two finite limit points of a binary lens (Rhie, 1995a). If $l=\left|x_{1}-x_{2}\right|$ denotes the separation between the lens masses,

$$
z_{* \pm}=x_{\mathrm{acm}} \pm i \sqrt{\epsilon_{1} \epsilon_{2}} l ; \quad x_{\mathrm{acm}} \equiv \epsilon_{1} x_{2}+\epsilon_{2} x_{1} .
$$

The limit points $z_{* \pm}$ are the images of $\omega_{* \pm}$ which we can calculate from the lens equation (2.1). If we let $\epsilon \equiv \epsilon_{1}-\epsilon_{2}$,

$$
\omega_{* \pm}=x_{\mathrm{acm}}-\frac{\epsilon}{l^{2}}\left(x_{2}-x_{1}\right) \pm i \sqrt{\epsilon_{1} \epsilon_{2}}\left(l-\frac{2}{l}\right)
$$

and $\omega_{*+}$ and $\omega_{*-}$ have to be degenerate in order to be one source producing two images at the limit points.

$$
\omega_{*+}=\omega_{*-} \quad \Rightarrow \quad l=\sqrt{2} \quad ; \quad \omega_{*}=\frac{1}{2}\left(x_{1}+x_{2}\right)
$$

In other words, a family of binary lenses with seperation $l=\sqrt{2}$ accommodate a source position with the infimum amplification 3 inside a caustic and the source position is at the mid-point of the lens positions irrespectively of the mass ratio that parameterizes the family.

The fact that there are two positive images implies that the source in (2.6) is inside a caustic. (In a binary lensing, the number of negative images is always bigger than that of positive images by one.) The three negative images are on the lens axis and all have $J=-3$. If the source is at the origin $\left(x_{1}+x_{2}=0\right)$, the lens equation for the negative images (on the real axis) is given by

$$
0=x\left(x^{2}-\frac{3}{2}\right)+\frac{\epsilon}{\sqrt{2}},
$$

where $x_{2}>x_{1}$ is assumed without loss of generality. The Jacobian determinant is given by

$$
J=1-\left(\frac{\left(x-x_{\mathrm{acm}}\right)^{2}+\epsilon_{1} \epsilon_{2} l^{2}}{\left(x-x_{1}\right)^{2}\left(x-x_{2}\right)^{2}}\right)^{2} \equiv 1-|\kappa|^{2} .
$$

When the lens is symmetric $(\epsilon=0)$, the images are at $x=0, \pm \sqrt{3 / 2}$ and it is easy to verify that $J=-3$ for each image. For an arbitrary $\epsilon$, one can reduce the 
numerator and denominator of $|\kappa|^{2}$ into second order polynomials using (2.7) to find that $|\kappa|^{2}=4$ nd so $J=-3$. The family of lenses defined by $l=\sqrt{2}$ have caustics consisting of either one connected loop or two. (For a three-loop caustic, $l<1$. See Rhie, 1995a). Two-loop caustics occur when the minor mass is small, and the source position for the infimum 3 is in the caustic near the minor mass. Inside the caustic (very) near the dominant mass, the amplification goes to infinity as the mass asymmetry grows because the caustic approaches that of a single lens.

Here we demonstrate that (1.1) does not hold when the source is outsidethe non-maximal domain of a binary lens - except when the source is at infinity. Let's consider a source located at the mid-point of a symmetric lens. When $l<2$, the source lies inside the caustic, and there are five images: two off the axis $\left(z= \pm \sqrt{d^{2}-1} ; d=l / 2\right)$, and the other three $\left(z=0, \pm \sqrt{d^{2}+1}\right)$ on the axis. As the separation $l$ grows beyond the bifurcation condition $l=2$, the caustic splits into two closed loops and the source falls in the outside domain. Thus, two out of the five images should disappear, and they are the two solutions $z= \pm \sqrt{d^{2}-1}$, which become real at $l=2$. Both have $J=-4 d^{2}\left(d^{2}-1\right)(<0$ for $l>2)$. Therefore, the other three images have $\sum_{\text {images }} J^{-1}>1(\rightarrow 1$ as $d \rightarrow \infty)$. Of course, the total amplification of the three images of a source outside can be dominated by negative images and $\sum_{\text {images }} J^{-1}<1$. An example is a source near the off-axis cusps which appear when the caustic bifurcates into three loops.

\section{3. $\sum J^{-1}=1$ for the Maximum Number of Images}

The lens equation (2.1) is a mapping from an image position to a source position: $z, \bar{z} \mapsto \omega$, and lensing is the inverse of the lens mapping: $\omega, \bar{\omega} \mapsto z$ which is either triple-valued or quintuple-valued. If we let $z_{1} \equiv z-x_{1}, z_{2} \equiv z-x_{2}$ and $z_{\circ} \equiv z-x_{\text {acm }}$, the binary lens equation looks quite simple.

$$
\omega=z-\frac{\bar{z}_{\circ}}{\bar{z}_{1} \bar{z}_{2}} \equiv z-f(\bar{z})
$$

We note that the effect of the masses $\epsilon_{j}: j=1,2$ is coupled only to the conjugate variable $\bar{z}$ and the relation between $\omega$ and $z$ is universal. We will see below that this universality is the underlying reason for the constraint (1.1), and thus, it holds not only for binary lenses but also for arbitrary $n$-point lenses.

In order to find the inverse function $z(\omega, \bar{\omega})$, we replace $\bar{z}$ in $(3.1)$ by $\bar{\omega}+f(z)$ : $\omega=z-f(\bar{\omega}+f(z))$ to find that $z$ satisfies a fifth order polynomial equation, 
$0=g(z ; \omega, \bar{\omega})$ (Witt, 1990). If $\omega_{j} ; j=0,1,2$ is defined similarly to $z_{j}$,

$$
g(z ; \omega, \bar{\omega})=(z-\omega)\left(z_{\circ}+\bar{\omega}_{1} z_{1} z_{2}\right)\left(z_{\circ}+\bar{\omega}_{2} z_{1} z_{2}\right)-z_{1} z_{2}\left(z_{\circ}+\bar{\omega}_{\circ} z_{1} z_{2}\right) .
$$

$g=0$ is an analytic equation in $z$ and always has five solutions. Thus, $g=0$ is not equivalent to the original lens equation when the source is outside where there are only three images. However, in the inside domains we are interested in here, the sources result in the maximum number of images 5 and so we can consider $0=g$ as the lens equation. If $a_{j}(\omega, \bar{\omega}) ; j=1,5$ are the zeros of $g$, we can factorize $g$ as $g(z ; \omega, \bar{\omega})=a_{\circ} \Pi_{j=1}^{5}\left(z-a_{j}\right)$, where $a_{\circ}=\bar{\omega}_{1} \bar{\omega}_{2}$. Now, we note from (2.3) that $J^{-1}$ is a very primary quantity we can calculate easily. The value of $J^{-1}$ - the amplification combined with the parity - at an image position $a_{j}$ is

$$
J^{-1}\left(a_{j}\right)=\partial_{\omega} z\left(a_{j}\right)=-\frac{\partial_{\omega} g}{\partial_{z} g}\left(a_{j}\right)=\partial_{\omega} a_{j}(\omega, \bar{\omega}) .
$$

By summing it over all the images using (3.2), we find the "sum rule" completing the proof.

$$
\sum_{j=1}^{5} J^{-1}\left(a_{j}\right)=\partial_{\omega} \sum_{j=1}^{5} a_{j}(\omega, \bar{\omega})=1 .
$$

The relevent terms in $g$ for the second equality are the highest order term for the whole polynomial and the next highest order term depending on $\omega:(z-\omega) z^{4} \bar{\omega}_{1} \bar{\omega}_{2}$. The constant 1 on the RHS is determined by the relation between $\omega$ and $z$ in the factor $(z-\omega)$.

For a single lens, there are two images and $g$ is a quadratic polynomial in $z$. Since $g=0$ is saturated by the images (or $g=0$ is equivalent to the lens equation), (3.4) holds for a single lens. Or, one can satisfy oneself by solving the quadratic lens equation and adding up $J^{-1}$ for the solutions. $\left(J=1-|z|^{-4}\right.$, where the lens is at $z=0$.)

Now, the generalization of (3.4) for an arbitrary class of $n$-point lens systems is only a matter of bookkeeping. The lens equation is given by $\omega=$ $z-\sum_{j=1}^{n} \epsilon_{j} / \bar{z}_{j} ; \quad \sum_{j=1}^{n} \epsilon_{j}=1$. We note that $f(\bar{z})$ in (3.1) is a quotient of a first order polynomial by a second order polynomial: $f=f_{1} / f_{2}$. For an $n$ point lens, $f(\bar{z})$ is a quotient of an $(n-1)$-th order polynomial by an $n$-th order polynomial: $f=f_{n-1} / f_{n}$. For a binary, the polynomial in $(3.2) g(z ; \omega, \bar{\omega}) \equiv$ $g_{2} \supset(z-\omega) \Pi_{j=1}^{2}\left(f_{1}+\bar{\omega}_{j} f_{2}\right) \supset a_{\circ}(z-\omega) z^{4}$. For an $n$-point lens, the corresponding polynomial $g(z ; \omega, \bar{\omega}) \equiv g_{n} \supset(z-\omega) \Pi_{j=1}^{n}\left(f_{n-1}+\bar{\omega}_{j} f_{n}\right) \quad \supset a_{\circ}(z-\omega) z^{n^{2}}$. 
Therefore,

$$
\sum_{j} J^{-1}\left(a_{j}\right)=\partial_{\omega} \sum_{j} a_{j}(\omega, \bar{\omega})=1 .
$$

We emphasize that the second equality of (3.5) holds only when the summation is over all the $n^{2}+1$ images, i.e., in the "maximal domains." Only in the "maximal domains", the lens equation is equivalent to $g=0$.

\section{Graded Caustic Domains}

There is no "inside a caustic" in a single lens $(n=1)$ because the caustic is point - a "collapsed caustic loop". For binary lenses $(n=2)$, "inside a caustic" is "a maximal domain" and the source plane consists of one, two or three "islands" of "maximal domains" surrounded by the outside. When $n \geq 3$, the caustics show more complicated structures as we have seen one of whose examples in figure 1. Now, how do we sort these apparently complicated structures and understand them intuitively? Actually, it is rather simple to see that a source plane consists of graded caustic domains - namely, domains with well-defined degrees. We only need to recall a couple of well-known facts. First, the number of images of a source remains the same if the source does not cross a caustic curve. So, we can spell out the self-evident definition of a domain: A domain of a source plane is an area bounded by but devoid of caustic curves. Second, the number of images changes by two - one positive and one negative - when a source crosses a caustic curve. Since caustic curves are oriented as determined by the phase angle of $\kappa$ we encountered in section 2 , we can consider the sign of caustic crossings. Then, given two randomly chosen domains, the algebraic sum of the number of caustic crossings with sign \pm is independent of the paths connecting the two domains (see Rhie, 1995a for more details). Now, define the degree of a caustic domain: The outside domain has degree zero and is denoted $\mathcal{D}^{\circ}$. If the algebraic sum of the caustic crossing between a domain and outside is $m$, the domain has degree $m$ and is denoted $\mathcal{D}^{m}$. (Lensing is a short range phenomenon, and $\mathcal{D}^{\circ}$ can consist of many domains where only one of them includes infinity.) Some of the corollaries are : 1) $\mathcal{D}^{m}$ is always nested in $\mathcal{D}^{m-1}$ 's and the number of images of a source in $\mathcal{D}^{m}$ is $n+1+2 m$. 2) A maximal domain is where a source has $n^{2}+1$ images by definition. Now, the number of images of a source in $\mathcal{D}^{\circ}$ is $n+1$ and thus the degree of a maximal domain is $\frac{n(n-1)}{2}$. If the maximum degree of the domains of an $n$-point lens is $N(n)$, the source plane is $\cup_{m=0}^{N(n)}\left\{\mathcal{D}^{m}\right\}$ where $N(n) \leq n(n-1) / 2$. We write $\sup N(n)=n(n-1) / 2$. 
Let's look at the example in figure 1: The source plane is made of one $\mathcal{D}^{\circ}$, nine $\mathcal{D}^{1}$ s, one $\mathcal{D}^{2}$ and one $\mathcal{D}^{3}$. As the seperation increases, the triple lens should converge to three single lenses: At a large separation, the source plane has three $\mathcal{D}^{1}$ 's near the lens positions. The point we are getting at is that the fact that $\mathcal{D}^{1}$ 's in the "ocean" of $\mathcal{D}^{\circ}$ is a generic feature of the source plane of an arbitrary $n$-point lens when the "correlations" of the lens masses is weak. As the masses come close and their "correlations" become strong, the $\mathcal{D}^{1}$ 's merge and generate higher hierarchical structures. In the case of binaries, however, $\mathcal{D}^{1}$ is also maximal and merging does not result in higher degrees.

There is only one positive image for a source at infinity and thus for a source anywhere in $\mathcal{D}^{\circ}$. Therefore, the number of positive images of a source in $\mathcal{D}^{m}$ is $1+m$, and the total amplification of the positive images is $A_{+}\left(\mathcal{D}^{m}\right) \geq 1+$ $m$. A universal constraint on negative images is that they have non-vanishing amplifications unless the source is at infinity $\in \mathcal{D}^{\circ}$. Therefore, $A_{\text {tot }}\left(\mathcal{D}^{m}\right)>1+m$ when $m \geq 1$ (or "inside the caustics"). Inside a 'maximal domain', the constraint (3.5) applies, and $A_{\text {tot }} \geq n(n-1)+1$. Whether this inequality is saturated is another question, and we will discuss this briefly below for $n \geq 3$.

$A_{\text {tot }}=n(n-1)+1$ only if all the positive images are at the limit points. Since there are $2(n-1)$ (finite) limit points, $n$ has to satisfy that $2(n-1)=$ $1+n(n-1) / 2$. In other words, $n=3$ is the only possibility besides the known cases of $n=1,2$. Indeed, we find that a source at the center of a symmetric ternary lens with separation (distance between two masses) $l=\sqrt[6]{2} \approx 1.12246$ has four positive images at the (finite) limit points. Therefore, $A_{+}=4$ and $A_{\text {tot }}=7$. It should be an interesting question whether the solution we have found is unique or one of many. For $n \geq 4$, the total amplification of the maximum number of images is formally $A_{\text {tot }}>n^{2}+1-n$. It should be interesting to investigate the significance of the high degree domains in terms of the size and frequency of the appearance in the source planes of multiple point lens systems.

Since this paper was submitted, the MACHO experiment has detected a binary event toward the LMC (Bennett et al., 1996, astro-ph/9606012) that shows the amplification between two caustic crossings less than 2. We have mentioned above that $A_{\text {tot }}>2$ inside any caustic of any $n$-point lens system. Therefore, one can definitely conclude that the event was "contaminated" by other than geometric effect due to gravity (such as the third component of the lens). If the "contamination" is due to blending of the source star that lies in the bar of the LMC, it may be verified by the centroid shift of the source star (MACHO, private communication). 


\section{ACKNOWLEDGEMENTS}

We thank D. Bennett for valuable input. This work was supported in part by the U.S. Department of Energy at the Lawrence Livermore National Laboratory under contract No. W-7405-Eng-48.

\section{REFERENCES}

Alard, C., Mao, S., and Guibert, J., 1995, submitted to A. \& A

Alcock, C., Akerlof, C.W., Allsman, R.A., Axelrod, T.S., Bennett, D.P., Chan, S., Cook, K.H., Freeman, K.C., Griest, K., Marshall, S.L., Park, H.-S., Perlmutter, S., Peterson, B.A., Pratt, M.R., Quinn, P.J., Rodgers, A.W., Stubbs, C.W., and Sutherland, W., 1993, Nature, 365, 621.

Alcock, C., Allsman, R.A., Axelrod, T.S., Bennett, D.P., Chan, S., Cook, K.H., Freeman, K.C., Griest, K., Marshall, S.L., Perlmutter, S., Peterson, B.A., Pratt, M.R., Quinn, P.J., Rodgers, A.W., Stubbs, C.W., and Sutherland, W., 1995, ApJ, in press.

Aubourg, E., Bareyre, P., Brehin, S., Gros, M., Lachieze-Rey, M., Laurent, B., Lesquoy, E., Magneville, C., Milsztajn, A., Moscosco, L., Queinnec, F., Rich, J., Spiro, M., Vigroux, L., Zylberajch, S., Ansari, R., Cavalier, F., Moniez, M., Beaulieu, J.-P., Ferlet, R., Grison, Ph., Vidal-Madjar, A., Guibert, J., Moreau, O., Tajahmady, F., Maurice, E., Prevot, L., and Gry, C., 1993, Nature, 365, 623.

Bennett, D.P. et al., AIP Conference Proceedings 336: Dark Matter, eds., S. S. Holt and C. L. Bennett, 1995, p.77.

Bourassa, R. R., Kantowski, R, Norton, T. D., 1973, ApJ, 185, 747.

Mao, S. and Paczyński, B., 1991, ApJ, 374, L37.

Rhie, S., 1995a, preprint.

Rhie, S., 1995b, preprint.

Tytler, D., et al., 1995, in preparation.

Udalski, A., Szymanski, M., Kaluzny, J., Kubiak, M., Krzeminski, W., Mateo, M., Preston, G.W., and Paczyński, B., 1993, Acta Astronomica 43, 289.

Udalski, A., Szymanski, M., Kaluzny, J., Kubiak, M., Krzeminski, W., Mateo, M., Preston, G.W., and Paczyński, B., 1994, Acta Astronomica, 44, 165.

Witt, H.J., 1990, A. \& A., 236, 311.

Witt, H. J. and Mao, S., 1995, ApJ, 447, L105. 


\section{FIGURE CAPTIONS}

1. The source plane of a symmetric ternary lens with the seperation (distance between two masses) $l=1$ : The caustics divide the source plane into a heirachy of "nested" domains. The domain marked by $\times$ is the maximal domain $\mathcal{D}^{3}$, and the number of images of a source in the area is 10 . Incidentally, $x$ is at the center of the lens positions. 


\section{APPENDIX}

We would like to appreciate many anonymous referees who have shown great interest in this paper since it was submitted a year ago. Since many of the issues are more general than is dealt with in this paper, we would like to include some of the discussions in this appendix starting with the ones more closely related to the content of this paper.

1. First, we show each step of the equations in (3.3) starting with the expression of the inverse Jacobian matrix in (2.2). The first step is a simple manipulation of the implicit function $g(z ; \omega, \bar{\omega})$ given in $(3.2)$. From $0=g(z ; \omega, \bar{\omega})$, we get

$$
0=d g(z ; \omega, \bar{\omega})=\partial_{z} g d z+\partial_{\omega} g d \omega+\partial_{\bar{\omega}} g d \bar{\omega},
$$

that is equivalent to

$$
d z=-\frac{\partial_{\omega} g}{\partial_{z} g} d \omega-\frac{\partial_{\bar{\omega}} g}{\partial_{z} g} d \bar{\omega}
$$

Therefore, we obtain the second equality of (3.3).

$$
\partial_{\omega} z=-\partial_{\omega} g / \partial_{z} g
$$

(This type of manipulations can be found in thermodynamics. Thermodynamic functions are determined by any two of the thermodynamic variables - density, pressure, temperature, entropy, etc - and the measurable quantities are partial derivatives of the thermodynamic functions.) One can calculate the coefficients (or the partial derivatives) $\partial_{\omega} g$ and $\partial_{z} g$ easily from the factorization of $g, g=$ $a_{\circ} \Pi_{j=1}^{5}\left(z-a_{j}\right)$ as given in the main text, where $a_{j}$ is a function of $\omega$ and $\bar{\omega}$, and $a_{\circ}=\bar{\omega}_{1} \bar{\omega}_{2}$ is independent of $\omega . \partial_{\omega} g\left(a_{j}\right)=a_{\circ}\left(-\partial_{\omega} a_{j}\right)$ and $\partial_{z} g\left(a_{j}\right)=a_{\circ}$ and one obtains the third equality.

2. We have shown that $A_{\text {tot }}=3$ can be achieved inside a caustic of a binary lens only when two positive images have amplification 1 and three negative images have amplification $1 / 3$. Having read through the proof, one can wonder, for example, why $A_{1}=1.2, A_{2}=1.1, A_{3}=0.2, A_{4}=0.3, A_{5}=0.2$ can not happen: Can we understand it more intuitively? We believe what is stated in the main text explains fairly obviously why the positive images have to have $A=1$ each and the total amplification of the negative images is 1: If $A_{+}$and $A_{-}$are the positive and negative total amplifications, they satisfy the following relations for $A_{\text {tot }}=3$.

$$
A_{+}+A_{-}=3 \quad ; \quad A_{+}-A_{-}=1,
$$

where the second equality is the sum rule $\sum_{\text {images }} J^{-1}=1$. Therefore, $A_{+}=2$ 
and $A_{-}=1$. Since the amplification of positive images are no less than 1 , each positive image must have amplification unity - in other words, they are the images falling on the two limit points. As is discussed in the main text, the fact that the source position has to be such that two positive images are at the limit points constrains the source position to be at the mid-point between the lens positions and the separation between the point lens elements to be $l=\sqrt{2}$. The class of two point lens systems is a two-parameter family given by $\{\epsilon, l\}$. The subclass of the two point lens systems which allows the infimum amplification $A_{\text {tot }}=3$ inside a caustic is a one-parameter family given by $\{\epsilon\}$ because of the constraint on the separation $l=\sqrt{2}$.

This leaves us with the rest of the question unanswered: Can we find an intuitive (physical or structural) reason why negative images all have the same amplification $1 / 3$ for $A_{\text {tot }}=3$ ? We are currently ignorant of an obvious reason, but we find it an interesting question to pursue. That is especially so because a similar condition holds for the case of the triple lens we discussed in section 4: The six negative images of the source with $A_{\text {tot }}=7$ all have the same amplification $1 / 2$.

3. Here we present the calculations involved in the example of the triple lens discussed in section 4 , which follows exactly the same line of analysis used for the case of binary lenses as described in the main text. The family of symmetric triple lenses is parameterized by the separation $l$. If the center of mass is at the origin, the lens positions can be given by $x_{j}=a \exp \left(\frac{i 2 \pi j}{3}\right) ; j=0,1,2$, where $a=l / \sqrt{3}$. Then the symmetric triple lens equation is

$$
\omega=z-\frac{\bar{z}^{2}}{\bar{z}^{3}-a^{3}} .
$$

The limit points are obtained by solving

$$
0=\kappa \equiv \partial \bar{\omega}=\frac{z^{4}+2 a^{3} z}{\left(z^{3}-a^{3}\right)^{2}} .
$$

In other words, there are four limit points.

$$
z_{*}=0, \quad \sqrt[3]{2} a e^{\frac{i \pi}{3}}, \quad-\sqrt[3]{2} a, \quad \sqrt[3]{2} a e^{\frac{i 5 \pi}{3}}
$$

Therefore, the source has to be at the origin, namely,

$$
\omega_{*}=0 \quad \Leftarrow \quad z_{*}=0
$$

Now, in order for the other three limit points $(\neq 0)$ to be the image positions of the same source, the only free parameter $l$ (or $a$ ) has to be fixed. Because of the 
3-fold symmetry of the lens system, we only need to consider one of them, say, the non-zero limit point on the real axis. From the lens equation (A1),

$$
0=z_{*}-\frac{z_{*}^{2}}{z_{*}^{3}-a^{3}} \quad \Rightarrow \quad l^{2}=\sqrt[3]{2} .
$$

Therefore, the symmetric triple lens $\left(\epsilon_{j}=1 / 3 ; j=1,2,3\right)$ with separation $l=$ $\sqrt[6]{2} \approx 1.12246$ accommodates a source position where all the positive images fall on the limit points (amplification unity). Applying the constraint of the sum rule in the maximal domain $\mathcal{D}^{3}$, one finds that the total amplification of the source at the origin is $A_{\text {tot }}=7 \quad\left(A_{-}=3\right)$ as is stated in the main text. Incidentally, the non-zero limit point on the real axis is $z_{*}=-\sqrt[3]{2} a=-\sqrt{2 / 3}$.

We might as well spell out the positions and amplifications of the negative images, which is a straightforward exercise one can carry out analytically. We note that the real lens equation (A2) has four real solutions. Two are the ones on the limit points we discussed above. The other two solutions satisfy the second order polynomial equation.

$$
0=z^{2}-\sqrt{\frac{2}{3}} z-\frac{1}{3} \quad \Rightarrow \quad z_{ \pm}=\frac{1}{\sqrt{6}} \pm \frac{1}{\sqrt{2}}
$$

Now, we can show that the Jacobian determinant at $z_{ \pm}$is $J_{ \pm}=-2$. We recall that

$$
J=1-|\kappa|^{2} ; \quad \kappa \equiv \partial \bar{\omega}=\frac{z^{4}+2 a^{3} z}{\left(z^{3}-a^{3}\right)^{2}} .
$$

Using the first equation in (A3), one can reduce $\kappa$ into a quotient of two linear order polynomials.

$$
\kappa=\frac{6 a^{3} z+1 / 3}{3 a^{3} z+1 / 3}
$$

Using the same reduction method, one can find that $\kappa^{2}=3$ and hence $J=-2$. Up to now, we have discussed only the negative images on the real axis. The other four negative image positions can be found by rotating these two solutions on the real axis by $2 \pi / 3$ and $4 \pi / 3$ with respect to the origin. In other words, the six negative images of the source at the center of mass are located at

$$
z=z_{ \pm}, \quad z_{ \pm} e^{\frac{i 2 \pi}{3}}, \quad z_{ \pm} e^{\frac{i 4 \pi}{3}}
$$

Now, the symmetry of the lens system guarantees that all the negative images have $J=-2$ (and hence $A=1 / 2$ ). 
4. The lens equation in complex coordinates is accepted well in the community, but the differentiation of the equation is not. However, the Jacobian determinant is one of the most important observable quantities in gravitational lensing, and one should know how to calculate it in whatever coordinate system one has chosen. We have chosen complex coordinates and hence it is most natural to examine the linear behavior of the lens equation in the same complex coordinates. The necessity to calculate the Jacobian determinant arises from that the relative brightness of an image of a lensed star is given by the ratio of the sizes of the image and its source star. In other words, we need to know what happens to an infinitesimal area element in the lens plane under the mapping given by the lens equation. (The lens equation is an explicit mapping from an image position to its source position.)

If we parameterize the lens plane by Cartesian coordinates $(x, y)$, the infinitesimal area element is given by $d x \wedge d y$, where $\wedge$ stands for the exterior product (or "cross product"): $d x \wedge d y=-d y \wedge d x$. (One must be familiar with "cross product" from fluid mechanics or electrodynamics.) In terms of the complex coordinates $(z, \bar{z})$, the area element (always real) is given by a multiple of $d z \wedge d \bar{z}$. We can calculate the multiplicity using $z=x+i y$ and $\bar{z}=x-i y$.

$$
d z \wedge d \bar{z}=(d x+i d y) \wedge(d x-i d y)=d x \wedge(-i d y)+i d y \wedge d x=-2 i d x \wedge d y .
$$

Therefore, the multiplicity is $i / 2$. Now, in order to compare the sizes of an infinitesimal image and its source, it suffices to calculate the ratio $d \omega \wedge d \bar{\omega} / d z \wedge d \bar{z}$ because the multiplicity $i / 2$ is a constant.

$$
d \omega \wedge d \bar{\omega}=(\partial \omega d z+\bar{\partial} \omega d \bar{z}) \wedge(\partial \bar{\omega} d z+\bar{\partial} \bar{\omega} d \bar{z})=(\partial \omega \bar{\partial} \bar{\omega}-\bar{\partial} \omega \partial \bar{\omega}) d z \wedge d \bar{z} .
$$

Therefore,

$$
\frac{d \omega \wedge d \bar{\omega}}{d z \wedge d \bar{z}}=|\partial \omega|^{2}-|\bar{\partial} \omega|^{2}
$$

It is well known in the community that the RHS is nothing but the Jacobian determinant $J=|\partial \omega|^{2}-|\bar{\partial} \omega|^{2}$. One can get this expression of $J$ in complex coordinates from the familiar expression of $J$ in the real Cartesian coordinates by replacing the coordinates by complex coordinates using $z=x+i y$ and $\bar{z}=x-i y$.

Jacobian determinant literally means determinant of Jacobian matrix. Where does the Jacobian matrix come into picture in the landscape of lensing? We recall that we need to know what happens to its source when an image changes 
its position slightly (or infinitesimally). In other words, we need to know the linear behavior of the lens equation.

$$
\begin{aligned}
& d \omega=\partial \omega d z+\bar{\partial} \omega d \bar{z} \\
& d \bar{\omega}=\partial \bar{\omega} d z+\bar{\partial} \bar{\omega} d \bar{z}
\end{aligned} .
$$

These coupled linear equations can be organized as follows.

$$
\left(\begin{array}{l}
d \omega \\
d \bar{\omega}
\end{array}\right)=\left(\begin{array}{ll}
\partial \omega & \bar{\partial} \omega \\
\partial \bar{\omega} & \bar{\partial} \bar{\omega}
\end{array}\right)\left(\begin{array}{l}
d z \\
d \bar{z}
\end{array}\right)
$$

where the usual matrix multiplication convention applies. This $(2 \times 2)$ matrix is called Jacobian matrix (to honor the historical development), and one can see that the determinant of the matrix gives rise to the expression of $J$ in (A4).

The equation (A6) shows clearly that $\partial_{z} \omega$ and $\partial_{\bar{z}} \omega$ in (2.2) and their complex conjugates constitute the Jacobian matrix elements in complex coordinate basis. However, this could mean a dilemma if one tries to recover the familiar Jacobian matrix in Cartesian coordinate basis by replacing the complex variables by the Cartesian coordinate variables because one is sure to fail! We mentioned above that exactly the same process works for the Jacobian determinant. So, what is wrong? Actually, there is nothing wrong. It's simply that Jacobian matrix "rotates" accordingly with the change of coordinate basis while Jacobian determinant remains invariant. Let's consider changing coordinates where the new coordinate basis is obtained by multiplying the old coordinate basis by a matrix $V$ from the left. For example,

$$
V\left(\begin{array}{l}
d \omega \\
d \bar{\omega}
\end{array}\right) \Leftarrow\left(\begin{array}{l}
d \omega \\
d \bar{\omega}
\end{array}\right) ; \quad V\left(\begin{array}{l}
d z \\
d \bar{z}
\end{array}\right) \Leftarrow\left(\begin{array}{l}
d z \\
d \bar{z}
\end{array}\right) .
$$

Now, we can rewrite (A6) in terms of the new basis.

$$
V\left(\begin{array}{l}
d \omega \\
d \bar{\omega}
\end{array}\right)=V\left(\begin{array}{ll}
\partial \omega & \bar{\partial} \omega \\
\partial \bar{\omega} & \bar{\partial} \bar{\omega}
\end{array}\right) V^{-1} V\left(\begin{array}{l}
d z \\
d \bar{z}
\end{array}\right)
$$

In other words, the Jacobian matrix "rotates" through a similarity transformation $\mathcal{J} \mapsto V \mathcal{J} V^{-1}$. If one tries to understand the Jacobian matrix in complex coordinates starting from the more familiar form in Cartesian coordinates, one should make sure to rotate the matrix as well as change the coordinate functions. Without the proper rotation carried out, one can erroneouly conclude that the 
matrix elements in (2.2) are wrong. On the other hand, the determinant of a matrix is invariant under a similarity transformation unlike the Jacobian matrix. In other words, the Jacobian determinant is a scalar and is coordinate independent. That shouldn't be surprising because the Jacobian determinant is an observable quantity. (Coordinate dependence of the Jacobian determinant would mean that the amplification of a star would change depending on which coordiante system we use to calculate it. That is undesirable.)

We might as well discuss why the inverse Jacobian matrix elements are given by (2.3). In order to take advantage of the Einstein summation convention, let's rename the complex variables as follows: $X_{1}=z, X_{2}=\bar{z}$ and $Y_{1}=\omega, Y_{2}=\bar{\omega}$. Then the Jacobian matrix elements of the lens equation are $\partial Y_{c} / \partial X_{a} ; a, c=1,2$. And the inverse Jacobian matrix elements are given by $\partial X_{b} / \partial Y_{c} ; c, b=1,2$ as one can easily check using the product rule $\sum_{c} \frac{\partial Y_{c}}{\partial X_{a}} \frac{\partial X_{b}}{\partial Y_{c}}=\delta_{a b}$. In other words, $\partial_{\omega} z$ and $\partial_{\bar{\omega}} z$ in (2.3) and their complex conjugates constitute the inverse Jacobian matrix elements. (In case one is not familiar with the product rule, see the formula above (3.2.11) in S. Weinberg, 1972, "Gravitation and Cosmology", John Wiley \& Sons, Inc.)

5. There have been some concern whether it is proper to denote the Jacobian determinant by $J$ or something like $\operatorname{det} J$ should be used where $J$ denotes the Jacobian matrix. In our opinion, as is reflected in our literary work here, the Jacobian determinant deserves a simple notation just as much as the amplification deserves a widely accepted simple notation $A$ or $\mu$. Jacobian determinant is a determinant mathematically and one can prefer a more explicit notation such as $\operatorname{det} J$. However, the importance of the determinant in lensing lies in that the determinant is a measurable quantity. The magnitude of the determinant is nothing but $1 / A$ and the sign of the determinant is the parity of the image. Furthermore, the determinant is a very important quantity in the understanding of the lens plane. In other words, the determinant $J$ plays a far more important role in lensing than is implied by the mere fact that the function is determinant of a matrix. As a minor comment, this is not the first occasion where the determinant is denoted by $J$. See Kayser et al., 1986, A\&A, 166, 36. (This paper contains a mistake in the definition of $J$, but it is an easily recognizable mistake one can not be confused by.) On the other hand, we are not insisting that everyone in the lensing community should use the same notations as we do. That is because physics lies in the structure of the system indenpedently of which letter of which alphabet system is used for which variable.

6. We would like to direct the attention to the last paragraph in section 2. The original purpose of the example discussed in this paragraph is to show how 
the sum rule fails in a "non-maximal domain". However, one can also examine the behavior of the images at a cusp crossing. The merit of this example is that it is analytically solvable leaving no room for numerical artifacts and ambiguities. As the caustic bifurcates when $l=2$, two images disappear. When $l \rightarrow 2$ and the source nears the cusp, the amplification of these two images increases as $1 / \sqrt{a}$ where $a$ is the distance of the source from the cusp. These are all expected. However, what may not have been expected is that the disappearing images are both positive. Isn't it contradictory to our conventional wisdom that two images of opposite parity disappear (or appear) at a caustic crossing? Strictly speaking, yes. At a cusp crossing, the images that disappear together have the same parity, while the two disappearing images at a line caustic crossing have the opposite parities. On the other hand, at a cusp crossing, there is the third image that crosses the critical curve where the two images disappear. In other words, the third image changes the parity (from negative to positive here), and there is no net change of the total parity of the images. This third image is the one whose amplification increases as $1 / a$ as the source nears the cusp similarly to the images of a single lens. Therefore, the conventional wisdom should be modified to the correct theorem that the number of images change by two - or a multiple of two where the caustics self-intersect - and the total parity remains the same at a caustic crossing.

One can find an illustration of cusp crossing in figure 8 in Schneider and Weiss, 1986, A\&A 164, 237. (The curvatures of the critical curve and that of the "off-axis" image trajectory in figure 8 have the opposite signs, but they can also have the same sign depending on the lens parameters. At a cusp crossing on the lens axis, they always have the same sign.)

7. One of the most interesting issues seems to be the controvery over our identification of lensing as the inverse mapping of the lens equation (the bottom of page 3$)$.

Let's consider the lensing of a star by a black hole. The BH dictates the geometry of the space around it, and the light from the star to an observer (or us) follows the null geodesics (or optical paths) allowed by the geometry of the "curved" space. Lensing refers to the phenomenon that the starlight follows the null geodesics of the curved space instead of the optical path the light would follow were there not be the BH. Each null geodesic "delivers" light from the star to the observer in its own way, and the light arrived at the observer through a specific optical path is called an image. The observer can see as many images as the number of null geodesics between the target star and the observer. Therefore, lensing refers to the process forward in time, namely, the propagation of the 
light from the light source through the available null geodesics to corresponding images, and thus it is sensible to talk about lensing of a star. On the other hand, propagation of light along a given null geodesic is a time reversible process. For example, the observer can send the light back to the source star by putting a mirror perpendicular to the optical path at the position of the image. (Orthogonality is well defined in general relativity.) In other words, the process lensing has a well defined inverse process.

One can study the optical path lengths through time delay measurements. One can study the spin of the photons or collectively the polarization of the light in the images. Or, one can study the beams of the photons - propagation vectors (or angular positions) and sizes - arriving at the observer. The lens equation addresses the relation of the angular positions of the images and their source star. More specifically, the lens equation determines the position of the source star for a given image position. In other words, the lens equation is an explicit mapping describing the inverse process.

When we introduce a model (here the lens equation), the physical process (lensing here) must be identified as a mathematical process within the mathematical system. (If not, what is the use of the model?) So, what is lensing as a mathematical process within the mathematical system defined by the lens equation? The question boils down to whether the inverse process has well defined inverse. The lens equation is a projection map from the lens plane onto itself where three or five images are mapped into one source position. Therefore, the inverse of the projection map (inverse process) is the mapping from the lens plane to itself where a source position is mapped into multiple images. This multiplicity of the images requires more specific definition of the inverse: Given an image, the restricted mapping from the image to its source and back to the same image is a well defiend identity mapping. In other words, the lens equation is invertible when restricted to one given image. This type of invertibility is said to be locally invertible. In other words, the lens equation is locally invertible. But, not everywhere. The mapping can be restricted to one image only when the image is distinguishable from the other images of the same source. Where two images merge to disappear or split to appear - in other words, on the critical curve, the distinguishability fails. This singular behavior is manifested in the factor $1 / J$ in the inverse Jacobian matrix elements in (2.3). Therefore, the inversion property of the lens equation is correctly stated as follows. The lens equation is locally invertible except on the critical curve. We should note that the physically measurable quantities such as magnification and distortion of the images belong to the local behavior. 


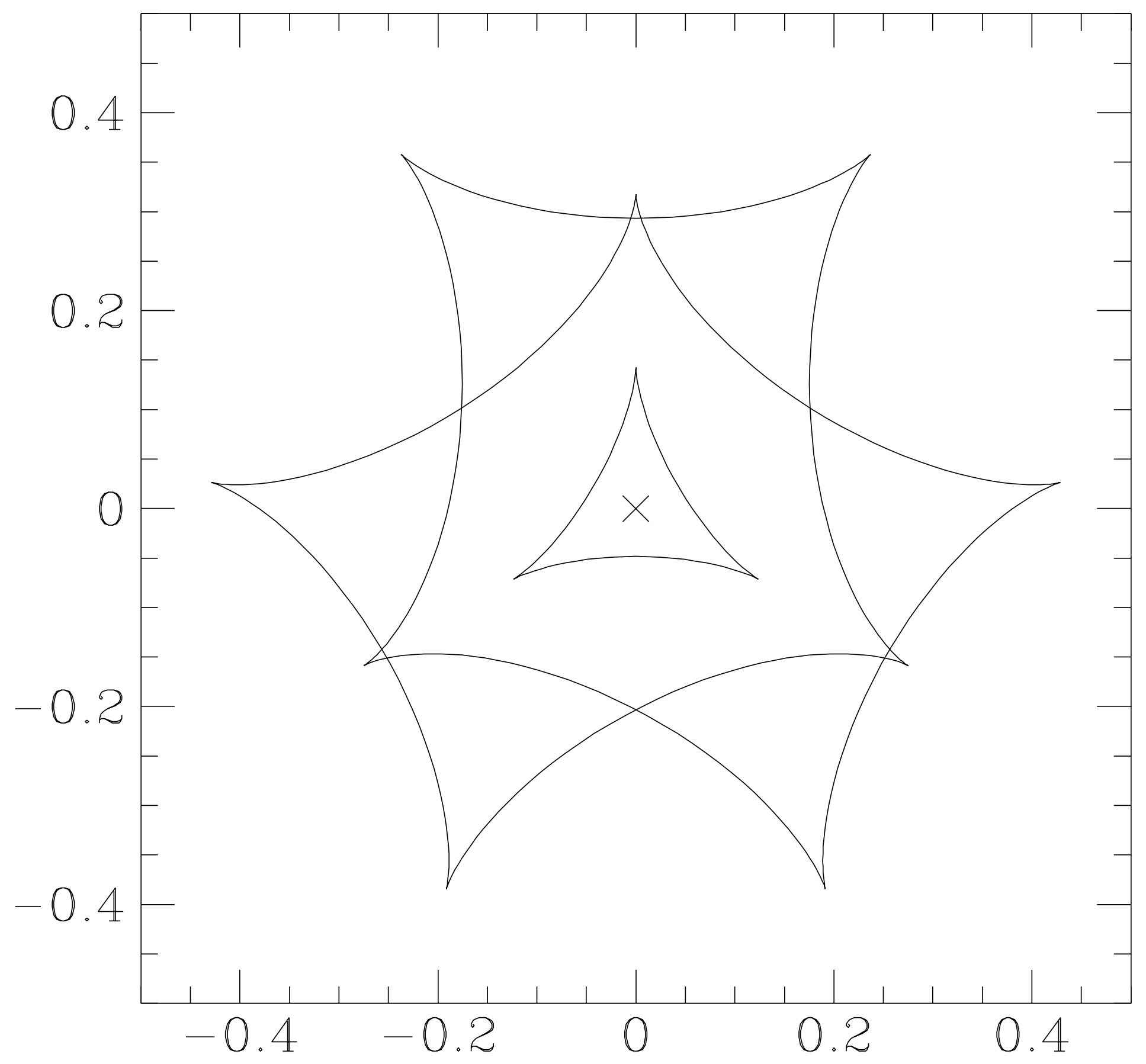

